International Journal of Robotic Computing

Vol. 2, No. 2 (2020) 1-20

(C) KS Press, Institute for Semantic Computing Foundation

DOI: $10.35708 / \mathrm{RC} 1869-126260$

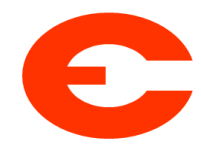

\title{
Identifying Hazardous Shapes in the Plane
}

\author{
Doron Nussbaum and Stephanie Thoumy \\ School of Computer Science \\ Carleton University \\ Ottawa, Ontario \\ nussbaum@scs . carleton. ca, StephanieThoumy@cmail. carleton. ca
}

Received (11/18/2018)

Revised (08/09/2019)

Accepted (01/20/2020)

\begin{abstract}
This paper explores the problem of identifying the shapes of invisible hazardous entities in $R^{2}$ by a set $S=\left\{s_{1}, s_{2}, \ldots, s_{k}\right\}$ of mobile sensors (autonomous robots). A hazardous entity, $H$, is a region that affects the operation of robots that either penetrate the area or come in contact with it. In this paper, we propose algorithms for searching a rectangular region for a stationary hazardous entity, where some a priori geometrical knowledge is given (e.g., edge size range), and if such an entity exists, then determine the area that it occupies. We explore entities that are convex in nature such as line segment, circles (discs), and simple convex shapes. The objectives are to minimize the distance travelled by the robots during the search phase, and to minimize the number of robots that are required to identify the region covered by the hazardous entity. The number of robots required to locate $H$ is three or four robots when $H$ is a line segment, two or three robots when $H$ is a circle, and seven robots are sufficient when $H$ is a triangle. Our results extend to $n$-vertex convex shapes and we show that $2 n+1$ robots are sufficient to determine the coverage of $H$.
\end{abstract}

\section{Introduction}

Mobile robots are gaining popularity especially in areas where the work is mundane and repetitive (e.g., cleaning) or when the environment is hazardous. In the latter case the hazardous area may not be known and must be correctly identified and marked either for clean-up operations or for avoidance by others.

In this paper we explore the problem of identifying an invisible hazardous entity, which is a polygonal or geometric shape in $2 \mathrm{D}$, by a set of autonomous robots. The hazardous entity, termed Hazardous Region (HR), is toxic and a 
robot that comes in contact with it ceases to operate (last known location can be transmitted). In this model the robots are point-robots that are capable of sensing the environment and of communicating with other robots. In particular, they can obtain the paths travelled by other robots and sense whether a robot has ceased to operate due to interaction with a HR (capable of obtaining the exact location of such event). By using the last known location of every robot that ceased to operate, the remaining robots determine the location and shape of the HR (preliminary results were presented in [9]).

The theoretical results presented in this paper have practical implications in studying path planning by robots for search and rescue, and in using robots in hazardous areas such as mine fields and nuclear disasters. Last, the solution presented here can be used to develop algorithmic solutions and strategies for border guarding and patrolling from intruders by robots (e.g., reducing the number of robots required to complete related tasks).

A more formal definition is the following: Given a 2D region $P$, an $\mathrm{HR} H$, and a set of point robots $S=\left\{s_{1}, s_{2}, \ldots, s_{k}\right\}$, can the set $S: 1$. determine whether $H \subset P$, and, 2. if $H \subset P$ then determine the location and coverage of $H$ in $P$.

Here we are looking at a number of optimization criteria: 1 . Reducing or minimizing the size of $S$. 2. Minimizing the distance travelled by the search party in order to determine the existence of $H$. Namely, determine $\min \left(\sum_{i=1}^{k} d\left(s_{i}\right)\right)$ where $d\left(s_{i}\right)$ is the distance that robot $s_{i}$ travelled. In doing so, provide the search path.

We make the following assumptions, which bound the size and shape of $P$ : 1. The relationship between $P$ and $H$ are as follows: either $H \cap P=\emptyset$ or $H \cap P=$ $H$.

2. $H \subset P$

3. The geometry of $H$ is somewhat restricted. Namely, $m_{1} \leq \operatorname{geom}(H) \leq m_{2}$ where $m_{1}$, and $m_{2}$ are constants and $\operatorname{geom}(H)$ is a geometrical property of $H$, such as area, length or diameter. For example, if $H$ is a triangle then each edge $e \in H$ has the following constraint $m_{1} \leq|e| \leq m_{2}$ where $m_{1}$, and $m_{2}$ are constants and $|e|$ is the length of edge $e$.

In the rest of the paper we assume that $P$ is a rectangular region and, when needed, use the notation $P=\left\{\left(x_{1}, y_{1}\right),\left(x_{2}, y_{2}\right)\right\}$ where $\left(x_{1}, y_{1}\right)$ and $\left(x_{2}, y_{2}\right)$ are the bottom-left and top-right coordinates of $P$ respectively.

To our knowledge no research was carried out on HR detection and identification in the Euclidean 2D space by a set of robots. Identifying HR regions is somewhat related to guarding and searching problems to which a large amount of research was carried out from art gallery problems to pursuit evaders, to searching for intruders [1]710]. More recently border patrolling research is carried out where the task of a set of mobile robots is to guard the boundary of a region (e.g., a circle or a rectangle) so that no intruders can enter undetected [13]. Research on HR problems was carried out on graphs where a set of mobile agents, which can travel from node to node in a network, attempts to detect and isolate a node that was infected with a HR entity [45|6]. 
Earlier work on a similar problem was carried out by Cole and Yap who explored the problem of probing of convex shapes using finger probes [2]. Skiena [11] discusses a number of probing options that can be used to discover geometrical shapes. Cole and Yap showed that $3 n-1$ probes are necessary and that $3 n$ probes are sufficient for identifying a convex shape in $2 \mathrm{D}$ using finger probes. In their algorithm the probes are made from infinity (line stabbing). The lower bound is based on two assumptions: a. the convex object location was already found and b. a known point inside the object is known. Although, the discovery of a HR can be viewed as a finger probe, there are three differences between the result in this paper and the result in [2 211]: a. there is no assumption made that the object was already found; $b$. the motion of the robot can be from any direction and thus the probes are not from infinity; and c. the discovery of the HR does not guarantee knowledge of a point inside the object (e.g., the first discovered point on $H$ may be a vertex of $H$ ). The only assumption that is made in this paper is some knowledge on the edge size of $H$. We also show that when additional information is given (e.g., orientation), the number of required robots to identify $H$ can be reduced.

The paper is organized as follows. We present general search algorithms for HRs that are line segments in Section 2 followed by HRs that are circles and triangles (convex polygon) in Sections 3 and 4 respectively. We conclude with a summary of the findings and some open problems in Section 5 .

\section{Hazardous Object is a Line Segment}

In this section, we provide solutions when the HR is a line segment. Namely, we study the following problem: given a rectangular region $P$ in $R^{2}$ (Euclidean 2D space), a hazardous entity that is a line segment where $m_{1} \leq \operatorname{length}(H) \leq m_{2}$ and a set of robots $S=\left\{s_{1}, s_{2}, \ldots, s_{k}\right\}$ determine:

1. Determine whether $H \subset P$

2. If $H \subset P$, then determine the location, orientation and length of $H$ using $S$, 3. Find efficient path that the robots should take in order to identify $H$.

4. Minimize the number of robots required to locate and identify $H$.

When searching for a line segment, one must take into consideration the fact that $H$ has no area; therefore, detecting $H$, for the first time, must be done by reaching $H$ in a direction $\hat{v}$ where $|\hat{H} \cdot \hat{v}| \neq 1$ where "." is the dot product, $\hat{v}$ is the unit vector in the motion direction of a robot and $\hat{H}$ is a unit vector capturing the orientation of $H$.

The orientation of HR may or may not be known in advance. Additional knowledge about the orientation of the HR can greatly affect the efficiency of the search pattern and the number of robots required to identify $H$. Here we look at three cases of a-priori knowledge about the orientations of the HR:

1. Orientation of $H$ is known and it is $x$-axis parallel

2. Orientation of $H$ is known and it is either $x$-axis parallel or $y$-axis parallel

3. Orientation of $H$ is not known (arbitrary orientation) 


\subsection{Searching for a Line Segment}

$\boldsymbol{H}$ is parallel to the $\boldsymbol{x}$-axis In this section we present an algorithm for finding a HR that is a line segment. Here $H$ is parallel to the $x$-axis within a rectangular region $P=\left\{\left(x_{1}, y_{1}\right),\left(x_{2}, y_{2}\right)\right\}$. The dimensions of $P$ are $w=x_{2}-x_{1}$ and $h=y_{2}-y_{1}$. We look at the detection problem by a single robot $s_{1}$.

In order to detect whether $H \subset P$ a snake like search path is used. The snake like search path is created as follows: divide $P$ into $\frac{w}{m}$ vertical strips, $V S^{i}, 1 \leq i \leq\left\lfloor\frac{w}{m}\right\rfloor$, of size $m$. Without loss of generality assume that $w=\left\lfloor\frac{w}{m}\right\rfloor * m$. The robot then traverses the stripes along the internal boundaries of the strips from the left side of $P$ to the right as shown in Figure 1 . We denote by $\pi_{v}$ the vertical snake like search pattern. We first show that the HR $H$ can be found by searching $P$ along $\pi_{v}$. Here we use $m=m_{1}$ because it is the minimum size that $H$ can be.

Lemma 1. Given a HR $H$ that is a line segment parallel to the $x$-axis $(|H|=$ $m)$, a set $S=\left\{s_{1}\right\}$ and a rectangular region $P, s_{1}$ can detect whether $H \subset P$ by searching $P$ along $\pi_{v}$.

Proof. Proof is given by contradiction. Thus assume that $s_{1}$ is travelling along $\pi_{v}$ and that $s_{1}$ does not find $H$. Let $p$ and $q$ be the left and right endpoints of $H$, respectively. Let $V S^{k} \in P$ be a strip that contains $p$, with left and right walls at $x_{1}^{k}$ and $x_{2}^{k}$ respectively $\left(x_{2}^{k}-x_{1}^{k}=m\right)$.

There are two cases to consider:

Case $11 \leq k<\frac{w}{m}$ - in this case $x_{1}^{k} \leq p_{x}$ where $p_{x}$ and $p_{y}$ are the $x$ and $y$ -coordinates of $p$, respectively. Let $p^{*}$ be a point with coordinates $\left(p_{x}+d, p_{y}\right)$, where $d=m-\left(p_{x}-x_{1}^{k}\right)$, The point $p^{*} \in H$ because $0 \leq d \leq m$. On the other hand $p^{*}=\left(p_{x}+d, p_{y}\right)=\left(p_{x}+m-\left(p_{x}-x_{1}^{k}\right), p_{y}\right)=\left(x_{1}^{k}+m, p_{y}\right)=\left(x_{2}^{k}, p_{y}\right)$, and therefore $p^{*} \in \pi_{v} s$. As a result when $s_{1}$ travels along $\pi_{v}$ it will encounter $H$ contradicting the assumption.

Case $2 k=\frac{w}{m}$ - in this case $x_{1}^{k} \leq p_{x}<x_{2}^{k}$ and therefore because $(|H|=m)$ and $x_{2}^{k}-x_{1}^{k}=m$ and $H \in P$ we have $p=\left(x_{1}^{k}, p_{y}\right)$. As a result when $s_{1}$ travels along $\pi_{v}$ it will encounter $H$ contradicting the assumption.

Lemma 1 provides some insight about the minimum number of points that must be visited by $s_{1}$ along any cross section of that is parallel to the $x$-axis.

Corollary 1. Given a $H R H$ that is a line segment parallel to the $x$-axis $(|H|=$ $m)$, a set $S=\left\{s_{1}\right\}$ and a rectangular region $P$, for any cross section of $P$, parallel to the $x$-axis,

1. $s_{1}$ must visit at least $\max \left\{1, \frac{w}{m}-1\right\}$ points in order to detect whether $H \subset P$.

2. if $q_{y} \notin \pi_{v}$ is the only point that $s_{1}$ visit in leftmost strip then $s_{1}$ must visit at least $\frac{w}{m}-1$ additional points on the cross section in order to detect whether $H \subset P$.

Lemma 2. In each strip $V S^{i}, 1 \leq i \leq \frac{w}{m}$, a search robot, $s_{1}$, must visit, for each $y \in\left[y_{1}, y_{2}\right]$, at least one point $p(x, y)$ where $x \in\left[x_{1}^{i}, x_{2}^{i}\right]$, in order to determine whether $H \in V S^{i}$. 
Proof. Proof is given by contraction. Assuming that there is a $y^{*} \in\left[y_{1}^{i}, y_{2}^{i}\right]$ such $s_{1}$ did not visit any point $p\left(x, y^{*}\right), x \in\left[x_{1}^{i}, x_{2}^{i}\right]$ and that $s_{1}$ correctly reported whether $H \in V S^{i}$ or whether $H \notin V S^{i}$. There are two cases:

Case $1 s_{1}$ reported that $H \notin V S^{i}$ : Let $q_{1}$ and $q_{2}$ be the left and right endpoints of $H$, respectively. Position $H$ such that $q_{1}=\left(x_{1}^{i}, y^{*}\right)$ and $q_{r}=\left(x_{2}^{i}, y^{*}\right)$. Robot $s_{1}$ did not visit any point $p\left(x, y^{*}\right), x \in\left[x_{1}^{i}, x_{2}^{i}\right], s_{1}$ and therefore failed to detect $H$ contradicting the assumption $s_{1}$ correctly reported that $H \notin V S^{i}$.

Case $2 s_{1}$ reported that $H \in V S^{i}$ : Let $q_{l}$ and $q_{r}$ be the left and right endpoints of $H$, respectively. In this case $H$ is initially positioned in $V S^{i}$ such that $q_{1}=\left(x_{1}^{i}, y^{*}\right)$ and $q_{2}=\left(x_{2}^{i}, y^{*}\right)$. We remove $H$ from $V S^{i}$ before $s_{1}$ search. Even though $s_{1}$ may have visited all points $p(x, y), x \in\left[x_{1}^{i}, x_{2}^{i}\right], y \in\left[y_{1}^{i}, y_{2}^{i}\right], y \neq$ $y^{*}, s_{1}$ could not have detected $H$ since $H$ was removed from $V S^{i}$ contradicting the assumption $s_{1}$ correctly reported that $H \in V S^{i}$.

Corollary 2. Given a HR $H$ that is a line segment parallel to the $x$-axis $(|H|=$ $m)$, a set $S=\left\{s_{1}\right\}$ and a rectangular region $P$, which is divided into $\frac{w}{m}$ vertical strips. If $d\left(\pi\left(V S^{i}\right)\right)$ is the path length that $s_{1}$ takes when searching for $H$ in $V S^{i}$ then $d\left(\pi\left(V S^{i}\right)\right) \geq\left|y_{2}-y_{1}\right|$.

Next, we show that vertical snake like pattern is optimal with respect to the travelled distance by $s_{1}$.

Lemma 3. Given a rectangular region $P=\left\{\left(x_{1}, y_{1}\right),\left(x_{2}, y_{2}\right)\right\}$, a line segment $H$ parallel to the $x$-axis as a HR, the snake like pattern, $\pi_{v}$ is optimal search pattern for a single robot.

Proof. A search robot must visit at least $\frac{w}{m}-1$ points, $\left\{p_{1}^{t}, \ldots, p_{\frac{w}{m}}^{t}-1\right\}$ and $\left\{p_{1}^{b}, \ldots, p_{\frac{w}{m}-1}^{b}\right\}$, on the top and bottom boundaries of $P$, respectively (Corollary 1.(1)). The search path is planar and does not self intersect (although parts of the path may meet at a point). By Corollary 1.(1), the robot should traverse the right boundary of the left most strip (otherwise the robot travels additional distance). By Corollary 1 (2), all other points in a cross section would line up on the boundary of the strips. This creates $\frac{w}{m}-1$ parallel vertical line segments, which must be connected because the robot travels continuously. The minimum distance to connect them is along the horizontal boundary of $P$ (e.g., using minimum spanning tree algorithm [8]).

The path length for detecting whether a HR that is a horizontal line segment is given in Equation 1

$$
\left|\pi_{v}\right|=\left(\frac{w}{m}-1\right) * h+w-2 * m_{1}
$$

$\boldsymbol{H}$ either parallel to the $\boldsymbol{x}$-axis or the $\boldsymbol{y}$-axis As shown in Section 2.1 when $H$ is parallel to the $x$-axis a search pattern $\pi_{v}$ is optimal. Similarly, when $H$ is parallel to the $y$-axis a search path pattern along the horizontal strips boundary, denoted by $\pi_{h}$, is optimal (Fig. 22). However, when $H$ is parallel to either $x$-axis or $y$-axis neither $\pi_{v}$ nor $\pi_{h}$ is sufficient because $H$ can always be 
embedded in $P$ by placing $H$ in a horizontal orientation if $\pi_{h}$ is used (vertical orientation if $\pi_{v}$ is used). In order to detect whether $P$ contains $H$ two search patterns are proposed: 1 . a grid like snake pattern is created by concatenating $\pi_{v}$ and $\pi_{h}$, denoted as $\pi_{v h}$ (Fig. 4), and 2. a diagonal snake pattern, denoted by $\pi_{d}$ (Fig. 3).

Grid Like Search Pattern The grid like search pattern. combines the vertical snake line pattern and the horizontal snake like search pattern into single search pattern by concatenating $\pi_{v}$ and $\pi_{h}$, denoted as $\pi_{v h}$ (Fig. 4).

The construction of the grid like pattern is done as follows:

- The strip width of $\pi_{v}$ is $m$ and the strip height of $\pi_{h}$ is $m$.

- Create a search path $\pi_{v}$ in $P$

- Create a search path $\pi_{h}$ in $P$

- Concatenate $\pi_{h}$ and $\pi_{v}$ at a corner of $P$ by traversing along a diagonal line from the end of one pattern and the start of the other pattern.

Lemma 4. Given a HR $H(|H|=m)$ as a line segment parallel to either the $x$-axis or the $x$-axis, a set $S=\left\{s_{1}\right\}$ and a rectangular region $P, S$ can detect $H$ in $P$ by searching $P$ along a grid like search pattern, $\pi_{v h}$.

Proof. Proof is similar to the proof of Lemma 1 and is therefore omitted.

The path length of $\pi_{v h}$ is given in Equation 2. Here we assume that $w=$ $\left\lfloor\frac{w}{m}\right\rfloor * m$, and that $h=\left\lfloor\frac{h}{m}\right\rfloor * m$ and that $w \geq h$.

$$
\begin{aligned}
\left|\pi_{v h}\right| & \left.=\left[\left(\frac{w}{m}-1\right) * \frac{h}{m}+\left(\frac{h}{m}-1\right) * \frac{w}{m}+\frac{w}{m}-2+\frac{h}{m}-2+\sqrt{2}\right)\right] * m \\
& \left.=\left[2 * \frac{w}{m} * \frac{h}{m}-4+\sqrt{2}\right)\right] * m \\
& =2 * \frac{w * h}{m}-4 m+\sqrt{2} * m
\end{aligned}
$$

Diagonal Search Path The diagonal search pattern $\pi_{d}$ is built on top the grid like search path (2.1). It is constructed as follows:

1. Find the height of the diagonal strip $m^{\prime}=\frac{m_{1}}{\sqrt{2}}$.

2. Create a grid like search path as described in Section 2.1

3. Create diagonal strips where the height of a strip is $m^{\prime}$ (Fig. 3)

Lemma 5. Given a HR $H$ as a line segment parallel to either the $x$-axis or the $y$-axis, a set $S=\left\{s_{1}\right\}$ and a rectangular region $P, S$ can detect $H$ in $P$ by searching $P$ along a diagonal snake path, $\pi_{d}$.

Proof. Proof is similar to the proof of Lemma 1 and is therefore omitted.

The path length of $\pi_{d}$ is given in Equation 3. Here we assume that $w=$ $\left\lfloor\frac{w}{m}\right\rfloor * m$, and that $h=\left\lfloor\frac{h}{m}\right\rfloor * m$ and that $w \geq h$.

$$
\begin{aligned}
\left|\pi_{d}\right| & =\left(\frac{w}{m} * \frac{h}{m} * \sqrt{2}\right) * m+\left(\frac{w}{m}+\frac{h}{m}-2\right) * m \\
& =\frac{w * h}{m} * \sqrt{2}+w+h-2 * m
\end{aligned}
$$


The search pattern lengths of $\pi_{d}$ and $\pi_{v h}$ depends on the shape of $P$. Namely, depending on the width and height of $P$ it is better to choose $\pi_{d}$ over $\pi_{v h}$. Equation 4 shows the conditions of choosing $\pi_{d}$ over $\pi_{v h}$.

$$
\begin{aligned}
\left|\pi_{v h}\right| & \stackrel{?}{\geq}\left|\pi_{d}\right| \\
2 * \frac{w * h}{m}-4 m+\sqrt{2} * m & \stackrel{?}{\geq} \frac{w * h}{m} * \sqrt{2}+w+h-2 * m \\
\frac{w * h}{m} *(2-\sqrt{2}) & \stackrel{?}{\geq} w+h+(2-\sqrt{2}) * m
\end{aligned}
$$

Orientation of $\boldsymbol{H}$ is not known In this section we look at HRs that are line segments with any orientation. When $H$ can exhibit any orientation no single snake like pattern can be used because $H$ can be embedded in $P$ with orientation that is parallel to the snake pattern. In order to overcome it a grid like snake pattern is used as in Section 2.1 (Fig. 4). However, in this case we are using $m^{\prime}$ for strip width of $\pi_{v}$ and the strip height of $\pi_{h}$ where $m^{\prime}=m_{1} * \cos (45)$. Note that the intersection of a vertical slab of $\pi_{v}$ and a horizontal slab of $\pi_{h}$ forms a square of size $m^{\prime} \mathrm{x} m^{\prime}$. As a result $H$ can be fully embedded in such a square only on the diagonal.

Lemma 6. Given a $H R H(|H|=m)$ as a line segment with arbitrary orientation, a set $S=\left\{s_{1}\right\}$ and a rectangular region $P, S$ can detect $H$ in $P$ by searching $P$ along a path $\pi_{v h}$.

Proof. Proof is similar to the proof of Lemma 1 and is therefore omitted.

The path length for detecting whether a HR with arbitrary orientation exists is given in Equation 5 It is the same as Equation 2 except that $m$ is replace with $m^{\prime}=m * \cos (45)$. Here we assume that $w=\left\lfloor\frac{w}{m^{\prime}}\right\rfloor * m^{\prime}$, and that $h=\left\lfloor\frac{h}{m^{\prime}}\right\rfloor * m^{\prime}$.

$$
\left|\pi_{v h}\right|=2 * \frac{w * h}{m^{\prime}}-4 m^{\prime}+\sqrt{2} * m^{\prime}
$$

\subsection{Identifying $H$}

Identifying $H$ is not a simple task since the length of $H$ is not known, rather only a range for $H$ is given. Once a $\mathrm{HR}$ is detected by a robot a single point on the line segment is known. What is left to determine are the two endpoints of $H$. Note, that here an assumption is made that the level of accuracy is such that the robots can travel in direction $\hat{H}$ and meet $H$ at its endpoint. Otherwise numerous robots must be used to identify a safe zone around $H$. In this case the number of robots will depend on the required accuracy by using binary search technique.

Determining the location of $H$ is carried out by sending additional robots to reach the two endpoints of the HR. When the orientation of $H$ is known two additional robots can detect the boundary of $H$ by moving in directions $\hat{H}$ and 


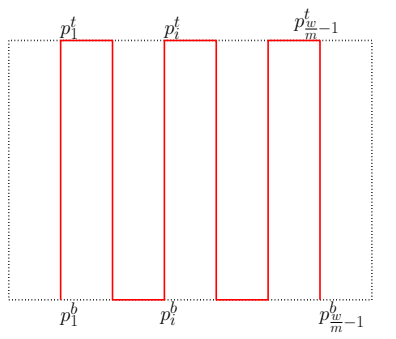

Fig. 1. Vertical snake like pattern used when a black hole is a horizontal line segment.

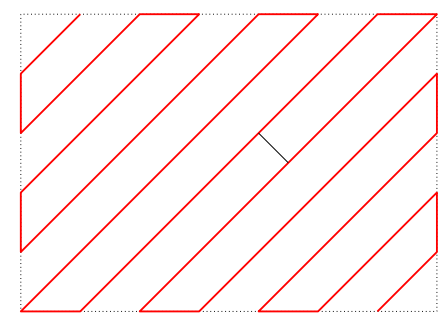

Fig. 3. Diagonal search pattern used when a black hole is either a vertical or horizontal line segment.

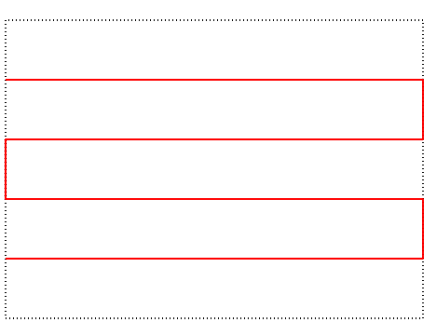

Fig. 2. Horizontal snake like pattern used when a black hole is a vertical line segment.

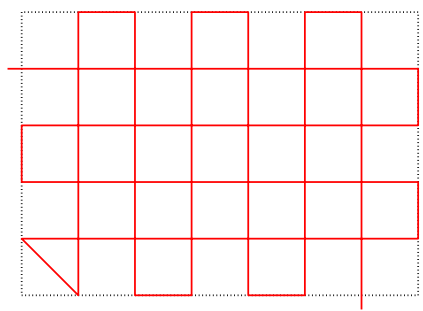

Fig. 4. Grid search pattern used when a black hole is either a vertical or horizontal line segment.

$-\hat{H}$, respectively. Let $q$ be the point where $H$ was detected. In this case one robot would move along the half line $q+s \hat{H}$ and the other along the half line $q-s \hat{H}$ ( $s$ is a scalar).

Lemma 7. Given a black hole $H$ that is a line segment parallel where $m_{1} \leq$ $|H| \leq m_{2}$, and that its existence was identified by a robot $s_{1}$, then

1. If $H$ is parallel to the $x$-axis then the region covered by $H$ can be determined by two additional robots.

2. If $H$ is parallel to the $y$-axis then the region covered by $H$ can be determined by two additional robots.

Proof. $1 . H$ is parallel to the $x$-axis - Let $q=(x, y)$ be the point where $s_{1}$ encountered $H$. Robot $s_{2}$ moves to point $q^{\prime}=\left(x-m_{2}-\epsilon, y\right)$. Robot $s_{2}$ can safely move to $q^{\prime}$ because $H$ cannot cover point $q^{\prime}$. Once $s_{2}$ reaches $q^{\prime}$ it moves in the direction $\overrightarrow{q^{\prime} q}$ until it encounters $H$ at point $q^{\prime \prime}=\left(x^{\prime \prime}, y\right)$. Robot $s_{2}$ will encounter $H$ because its path has the same $y$ coordinate as $H$. Robot $s_{3}$ can then safely move to position $q^{3}=\left(x^{\prime \prime}+m_{2}+\epsilon, y\right)$. Once $s_{3}$ has reached $q^{3}$ it moves in direction $\overrightarrow{q q^{\prime}}$ until it encounters the rightmost end of $H$.

2. $H$ is parallel to the $y$-axis - proof is similar to 1 and is therefore omitted. 
When the orientation is known to be either parallel to the $x$-axis or the $y$-axis, a second robot $s_{2}$ can determine the orientation and find one of the endpoints at the same time.

Lemma 8. Given a black hole $H$, where $m_{1} \leq|H| \leq m_{2}$, as a line segment parallel to the $x$-axis or the $y$-axisthat its location was identified by a robot $s_{1}$, the region covered by $H$ can be determined by two additional robots.

Proof. Let $q=(x, y)$ be the point where $s_{1}$ encountered $H$. Since $H$ can be parallel to either the $x$-axis or the $y$-axis, robot $s_{2}$ must a. determine the orientation of $H$ and b. determine one of the end points. Robot $s_{2}$ attempts to reach one of the endpoints by travelling around $q$ clockwise and exploring whether $H$ is horizontal or vertical. There are four cases to examine (Figure 5):

1. $H$ is horizontal and mostly lies to the left of $q$ - In this case $s_{2}$ attempts to reach the leftmost point of $H$ by moving to point $q^{1}=\left(x-m_{2}, y\right)$. It then moves along $\overrightarrow{q^{1} q}$ until it is a distance $\frac{m_{1}}{2}$ from $q$. If $H$ is horizontal and the majority of it lies to the left of $q$ then $s_{1}$ would find it because $m_{1} \leq|H| \leq m_{2}$. If $s_{1}$ did not encounter $H$ then either $H$ is vertical or the majority of it lies to the right of $q$. It then assumes that $H$ is vertical (Case 2 .

2. $H$ is vertical and mostly lies above $q$ - It then moves to point $q^{2}=$ $\left(x, y+m_{2}\right)$ to examine whether $H$ is vertical. It does so by moving along $\overrightarrow{q^{2} q}$ until it is $\frac{m_{1}}{2}$ from $q$. If $H$ is vertical and most of it lies above $q$ then it will find $H$ because $m_{1} \leq|H| \leq m_{2}$. Otherwise it continues to check if $H$ is horizontal and its majority lies to the right of $q$ (Case 3 ).

3. $H$ is horizontal and mostly lies to the right of $q-$ Robot $s_{1}$ moves to point $q^{3}=\left(x+m_{2}, y\right)$ and then start moving along $\overrightarrow{q^{3} q}$ until it is a distance $\frac{m_{1}}{2}$. If $H$ is horizontal then it must be found because most of it lies to the right of $q$. Otherwise if would have found it in Case1. If $H$ is not found then it must be vertical (Case 4).

4. $H$ is vertical and mostly lies below $q-$ Robot $s_{1}$ then moves to point $q^{4}=\left(x, y-m_{2}\right)$ and then moving along $\overrightarrow{q^{4} q}$ until it is a distance $\frac{m_{1}}{2}$. If $H$ is vertical then most of it lies below $q$ (otherwise $s_{1}$ would have found $H$ in Case 2).

When the orientation of $H$ is not known ( $H$ can have an arbitrary orientation) then the orientation of $H$ must first be determined.

Lemma 9. Given a $H R H$ that is a line segment, where $m_{1} \leq|H| \leq m_{2}$, as a line segment with arbitrary orientation that its location was identified by a robot $s_{1}$, the region covered by $H$ can be determined by three additional robots.

Proof. Let $q=(x, y)$ be the point where $s_{1}$ encountered $H$ and let $d_{\epsilon}<m_{1}$ be the distance between $s_{1}$ and $s_{2}$ that is required to determine the orientation of $H$. Robot $s_{2}$ moves towards point $q^{\prime}=\left(x-d_{\epsilon}, y\right)$. If $s_{2}$ encounters $H$ then 


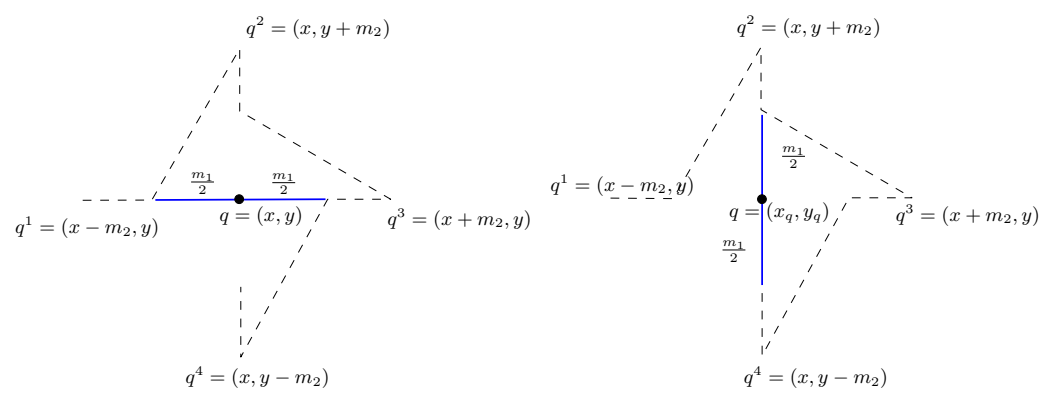

Fig. 5. Path of robot $s_{2}$ as it tries to determine whether $H$ is horizontal or vertical and to detect one of the endpoints of $H$..

the orientation can be determined. Otherwise, $s_{2}$ starts to move around $q$ in a circular path of radius $d_{\epsilon}$ until it encounters $H$ ( $s_{2}$ will encounter $H$ because $\left.d_{\epsilon}<m_{1}\right)$. Once the orientation is determined two additional robots, $s_{3}$ and $s_{4}$ are used to determine the endpoints of $H$ in a similar way to that in Lemma 7.

This leads to the following corollary when the orientation of $H$ is given.

Corollary 3. Given a $H R H$ that is a line segment, where $m_{1} \leq|H| \leq m_{2}$, with a known orientation the endpoints of $H$ can be determined by two additional robots.

Theorem 1 summarizes the results for identification. Note, that we did not discuss the distance travelled to identify $H$ because different strategies and different models can be used to do so. In our model of computation, we assume that the remaining robots in the set are at home base and are sent only when a HR is identified.

Theorem 1. Given a HR $H$ that is a line segment, where $m_{1} \leq|H| \leq m_{2}$, and that its existence was identified by a robot $s_{1}$, the region covered by $H$ can be determined by:

1. two additional robots if orientation of $H$ is known and is parallel to the $x$-axis

2. two additional robots if orientation of $H$ is known and is parallel to the $y$-axis

3. two additional robots if orientation of $H$ is known and is parallel to the $x$-axis or the $y$-axis

4. three additional robots if orientation of $H$ is not known and is arbitrary

\section{Circular Hazardous Object}

In this section we study the HR problem when $P$ is a rectangle and $H$ is a circle with a radius $r$, where $r_{\min } \leq r \leq r_{\max }$, and a set of sensors $S=\left\{s_{1}, s_{2}, \ldots, s_{k}\right\}$. 


\subsection{Searching for a Circular Object}

Similarly to Section 2.1 we first establish a path that must be traversed in order to determine whether $H$ exists. The path is geared towards a search by a single robot $s_{1}$. In the discussion on the construction of the path we assume that $H$ has a radius $r=r_{\text {min }}$.

Observation 2 Given a rectangular region $P=\left\{(0,0),\left(x_{2}, y_{2}\right)\right\}, 2 r \leq x_{2}, 2 r \leq$ $y_{2}$ and a circular $H R H$ that is tangent to the $x$-axis then a robot $s_{1}$ travelling along a path where each path point $(x, y), 0 \leq x \leq x_{2}, 0 \leq y \leq 2 r$ holds, will come in contact with $H$.

Based on Observation 2 we can establish a search path. Two possible search paths are: 1 . a modified spiral path, denoted by $\pi_{s^{\prime}}$, and 2 . a modified snake like path (either horizontal, $\pi_{h^{\prime}}$ or vertical $\pi_{v^{\prime}}$ ). The modified spiral path is a spiral path with the following properties: a. each corner of the path has an ear; $b$. the path does not touch the boundary of $P$ with the exception of the start point; c. the spacing between two adjacent parallel segments of the path is $2 r$; and d. the end of spiral is at distance $r$ from self intersecting the spiral path. Figure 6 shows a modified spiral path, which consists of ears and straight lines while Figure 7 shows the modified snake like pattern. Figure 8 shows the path layout and the construction of an ear. Note, that a circular HR is not limited to the drawn locations of the circles. Rather, the circles in the figures show possible locations of the HRs within $P$.

An ear is constructed as follows: each corner of the search path (either spiral or snake like) is extended to form an ear. Figure 8 shows an ear near the bottom left vertex of $P$. Without loss of generality assume that the bottom left vertex of $P$ is at $(0,0)$. The ear's apex is at coordinate $\left(\left(1+\frac{1}{\sqrt{2}}\right) r,\left(1+\frac{1}{\sqrt{2}}\right) r\right)$ and the two legs are at coordinates $(2 r, 3 r)$ and $(3 r, 2 r)$. A robot travelling along an ear will cover a distance of $(2 \sqrt{6-3 \sqrt{2}}) r$ (Equation 6).

$$
|e a r|=2 \sqrt{((1-\sin (45)) r)^{2}+((2-\cos (45)) r)^{2}}=2 r * \sqrt{6-3 \sqrt{2}}
$$

Lemma 10. Robot $s_{1}$ that searches $P$ for a circular $H R, H$, with radius $r$ along a modified spiral path, $\pi_{s^{\prime}}$, would find $H$, if $H$ exists in $P$.

Proof. The position of $H$ can either be along the boundary of $P$ or in the interior of $P$.

1. $H$ is positioned along the boundary of $P$ - When $s_{1}$ travels along the first four edges of the spiral path it is always at distance $2 r$ from the boundary of $P$. Thus, if only one edge of $P$ is tangent to $H$ then, based on Observation 2, $s_{1}$ will come in contact with $H$ or b travelling along the ear. If two edges of $P$ are tangent to $H$ then $H$ is positioned in a corner of $P$. In this case $s_{1}$ travelling along the ear will touch $H$ at the apex of the ear. 


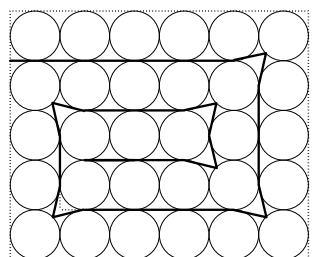

Fig. 6. Modified spiral search pattern. The circles demonstrate possible positions of $H$, thus assisting in computing the path

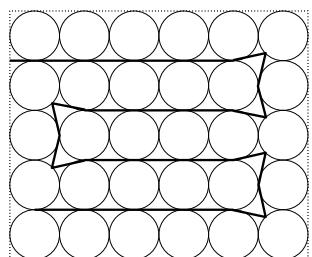

Fig. 7. Modified horizontal snake like search pattern. The circles demonstrate possible positions of $H$, thus assisting in computing the path

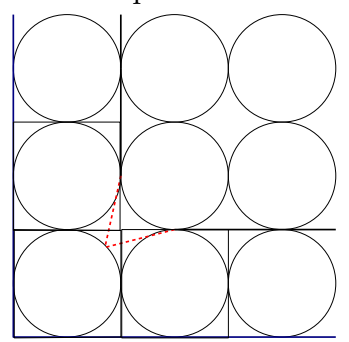

Fig. 8. The circles show the possible locations of the HRs within $P$ in order to demonstrate the path layout and the construction of an ear (the bottom left corner of $P$ shown in Figure 6. The thin line segments between the circles were drawn in order to demonstrate how the region can be divided into a grid. A search path (either a modified snake like or a modified spiral) would travel along the grid. Note, that a circular HR is not limited to the drawn locations of the circles.

2. $H$ is positioned in the interior of $P$ - in this case $H$ either intersects the spiral path, in which it will be detected, or $H$ is positioned between two sections of the path. In the latter case $H$ is positioned between an outer section and an inner section of the spiral path. The proof of this case is similar to Case 1 and is omitted.

A similar construction is carried out for a horizontal modified snake like path, denoted by $\pi_{h}^{\prime}$ (Fig. 7). Path, $\pi_{h}^{\prime}$ is constructed with the following modifications: a. each corner of the path is transformed to an ear; b. the path does not reach the boundary of $P$ with the exception of the start point; c. the path is constructed along strips of width $2 r$; and $d$. the end of the path is at distance $r$ from intersecting the boundary of $P$. As in the case of the spiral path, a robot $s_{1}$ travelling along a snake like path will find $H$ if it is in $P$.

Lemma 11. Robot $s_{1}$ that searches $P$ for a circular $H R, H$, with radius $r$ along a modified vertical path or modified horizontal path would find $H$, if $H$ exists in $P$.

Proof. Proof is similar to that of Lemma 10 and is therefore omitted.

The two path types, $\pi_{h^{\prime}}\left(\pi_{v^{\prime}}\right)$ and $\pi_{s^{\prime}}$ are equally good at detecting $H$ if $H$ exists. This raises the question "which path type should the search team use?" 
The answer depends on the comparison criteria. Here we examine the following criteria: 1. path length; 2. path start and end location; 3. relation of search path to boundary of $P$; and 4 . suitability for multiple search robots.

Path Length - This is the main criterion because it greatly affects the search robot with respect to the amount of time as well as the amount of energy required to search for $H$. The path length of the two schemes is the same as shown in Equations 7 and 9 below.

The snake like path direction, vertical or horizontal, is determined by the dimensions of $P$. Namely, if $w \geq h$ then $\pi_{h^{\prime}}$ is used, otherwise $\pi_{v^{\prime}}$ is used. Here we assume that $w \geq h$ and therefore $\pi_{h^{\prime}}$ is used (Fig. 7). The path length is given in Eq. 7. Here we assume that $w=\left\lfloor\frac{w}{2 r}\right\rfloor * 2 r$, and that $h=\left\lfloor\frac{h}{2 r}\right\rfloor * 2 r$.

$$
\begin{aligned}
\left|\pi_{h^{\prime}}\right| & =\left[\left(\frac{w}{2 r}-3\right)\left(\frac{h}{2 r}-1\right)\right] 2 r+5 r+\left[2+2\left(\frac{h}{2 r}-3\right)\right] \mid \text { ear } \mid \\
& =\left[\left(\frac{w}{2 r}-3\right)\left(\frac{h}{2 r}-1\right)\right] 2 r+5 r+\left[2\left(\frac{h}{2 r}-2\right)\right] \mid \text { ear } \mid \\
& =\frac{w h}{2 r}-3 h-w+11 r+\left[2\left(\frac{h}{2 r}-2\right)\right] \mid \text { ear } \mid
\end{aligned}
$$

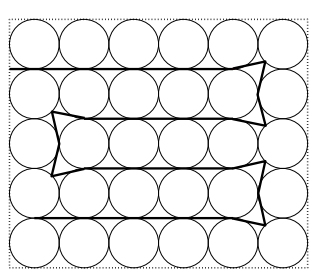

Fig. 9. Modified horizontal snake like search pattern. The circles are drawn as possible positions of $H$ and to assist in computing the path length.

When $w<h$ a modified horizontal snake path, $\pi_{v^{\prime}}$ should be selected. This can be seen from the following computation that compares $\left|\pi_{h^{\prime}}\right|$ and $\left|\pi_{v^{\prime}}\right|$ (Equation 8p. Here we assume that $w \geq h$. Note, that when computing $\left|\pi_{v^{\prime}}\right|$ the roles of $w$ and $h$ are reversed.

$$
\begin{aligned}
\left|\pi_{h^{\prime}}\right| & \stackrel{?}{\leq}\left|\pi_{v^{\prime}}\right| \\
\frac{w h}{2 r}-3 h-w+11 r+\left[2\left(\frac{h}{2 r}-2\right)\right] \mid \text { ear } \mid & \stackrel{?}{\leq} \frac{w h}{2 r}-3 w-h+11 r+\left[2\left(\frac{w}{2 r}-2\right)\right] \mid \text { ear } \mid \\
2 w-\left[2 \frac{w}{2 r}-4\right] \mid \text { ear } \mid & \stackrel{?}{\leq} 2 h-\left[2 \frac{h}{2 r}-4\right] \mid \text { ear } \mid \\
w\left(1-\frac{\mid \text { ear } \mid}{2 r}\right) & \stackrel{?}{\leq} h\left(1-\frac{\mid \text { ear } \mid}{2 r}\right)
\end{aligned}
$$


Equation 8 is true only if $w \leq h$ because $\left(1-\frac{|e a r|}{2 r} \leq 0\right)$.

Similar calculation is carried out for the modified spiral path. Here the straight line segments consist of vertical line segments and horizontal line segments. Note, that when $w \geq h$ the first leg of the spiral path is horizontal.

$$
\begin{aligned}
\left|\pi_{s^{\prime}}\right|= & (\# \text { horizontal }) * 2 r+(\# \text { vertical }) * 2 r+r+(\# \text { ears }) * \mid \text { ear } \mid \\
= & {\left[\frac{\left(\frac{h}{2 r}-2\right)\left(2 \frac{w}{2 r}-\frac{h}{2 r}-1\right)}{2}+\left(\frac{w}{2 r}-\frac{h}{2 r}+1\right)\right] * 2 r+} \\
& {\left[\frac{\left(\frac{h}{2 r}-2\right)\left(\frac{h}{2 r}-3\right)}{2}\right] * 2 r+r+4\left[\frac{\frac{h}{2 r}-2}{2}\right] \mid \text { ear } \mid } \\
= & {\left[\frac{2 \frac{w h}{4 r^{2}}-2 \frac{w}{2 r}-6 \frac{h}{2 r}+10}{2}\right] * 2 r+r+2\left(\frac{h}{2 r}-2\right) \mid \text { ear } \mid } \\
= & {\left[\frac{w h}{4 r^{2}}-\frac{w}{2 r}-3 \frac{h}{2 r}+5\right] * 2 r+r+2\left(\frac{h}{2 r}-2\right) \mid \text { ear } \mid } \\
= & \frac{w h}{2 r}-w-3 h+11 r+2\left(\frac{h}{2 r}-2\right) \mid \text { ear } \mid
\end{aligned}
$$

Since the path length is the same the choice of search scheme will depend only on subjective criteria and not on objective criterion (path length). However, based on the subjective criteria, (path start and end locations, relation of search path boundary of $P$ and suitability for multiple robot search) it seems that the modified snake like path may, in general, be better:

Path Start and End Locations - this criterion examines the effects of unsuccessful search ( $H$ was not found) by the search robot. Both schemes start from a location on the boundary that is at distance $2 r$ from a corner of $P$. However, the termination point of the search paths is significantly different. The end point of $\pi_{s^{\prime}}$ is roughly at the centre of $P$ while the end point of $\pi_{h^{\prime}}$ is at distance $r$ from the boundary of $P$. The snake like pattern is better if $s_{1}$ is required to leave $P$ immediately where the spiral like pattern is better if $s_{1}$ is required to return to the start point. If $P$ is divided into regions then the choice of pattern may depend on the dimensions of $P$ and the regions search order.

Relation of Search Path to the Boundary of $P$ - this criterion looks at the isolation of the boundary of $P$ by the search path. This is advantageous when $P$ is divided into smaller regions. The isolation of the boundary of $H$ can provide a safe path to all other regions so that they can be searched simultaneously. The spiral path seems to have an advantage because it scans the boundary of $P$ first and therefore isolates the search area.

Suitability for Multiple Search Robots - this criterion looks at the ease of which the path can be modified for using multiple robots to search $P$. Here the snake like path has clear advantage here. The shape of the path can be easily partitioned into subsections allowing multiple robots to traverse the complete search path. 


\section{$3.2 \quad$ Identifying $H$}

Identifying $H$ is a relatively easy task because a circle is a symmetric shape from all orientations. Depending on the information that is available about $H$ either two or three additional robots are required to identify $H$ as shown in Lemma 12 .

Lemma 12. Given a circular $H R H$ that was detected by a robot $s_{1}, H$ can be identified by:

1. One additional robot if the exact radius of $H$ is known

2. Two additional robots if the radius of $H$ has a given range

Proof. Proof is omitted due to simplicity.

\section{Triangular Hazardous Object}

In this section, we study the HR problem when $H$ is a triangle. When dealing with triangles as HRs, then additional information about the geometry of the triangle can be exploited to determine the search path and/or to reduce the number of robots that are required to detect and identify the area covered by $H$. Examples of additional geometrical knowledge are: triangular shapes such as equilateral, right-angle, isosceles, etc.; and the level of fatness (ratio between perimeter and area). However, in this paper, no knowledge about the geometry of $H$ is used, other than the restriction on the size of its edges denoted by $e_{1}, e_{2}$ and $e_{3}$. Namely, $m_{1} \leq\left|e_{i}\right| \leq m_{2}, 1 \leq i \leq 3$.

\subsection{Searching for Triangular Object}

Searching for $H$ is carried out in a similar way to that presented in Section 2.1 when the orientation of the line segment is not known. Namely, search path $\pi_{v h}$ is used to determine whether $H$ exists. The robots need to determine what is the width of the vertical slabs and the height of the horizontal slabs of the search path. The smallest triangle that meets the geometric constraints is an equilateral triangle where each edge size is $m_{1}$.

Observation 3 The width of the vertical slabs and the height of the horizontal slabs is determined by the size of the smallest square that can embed an equilateral triangle where the edge size is $m_{1}$.

Figure 10 shows an embedding of an equilateral triangle, where the edges size if $m_{1}$ into a square. What is left to determine is the size of angle $\alpha$.

Lemma 13. Given an equilateral triangle, $T$ with edge size of $d$ the smallest square that can contain $T$ is $d * \cos \left(15^{\circ}\right) X d * \cos \left(15^{\circ}\right)$.

Proof. Let $e$ be an edge of the containing square. The size of $e$ depends on angles $\alpha$ and $\beta$, namely, $|e|=\min (d * \cos (\alpha), d * \cos (\beta))$. The minimum size of $e$ occur when $\alpha=\beta$ since $\alpha \leq \beta \Longrightarrow d * \cos (\alpha) \geq d * \cos (\beta))$ and $\alpha \geq \beta \Longrightarrow$ $d * \cos (\alpha) \leq d * \cos (\beta))$.

Therefore $2 \alpha+60=90 \Longrightarrow \alpha=15$ 


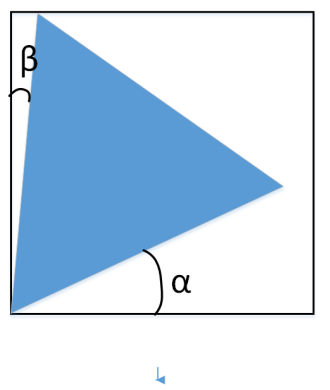

Fig. 10. embedding an equilateral triangle inside a square

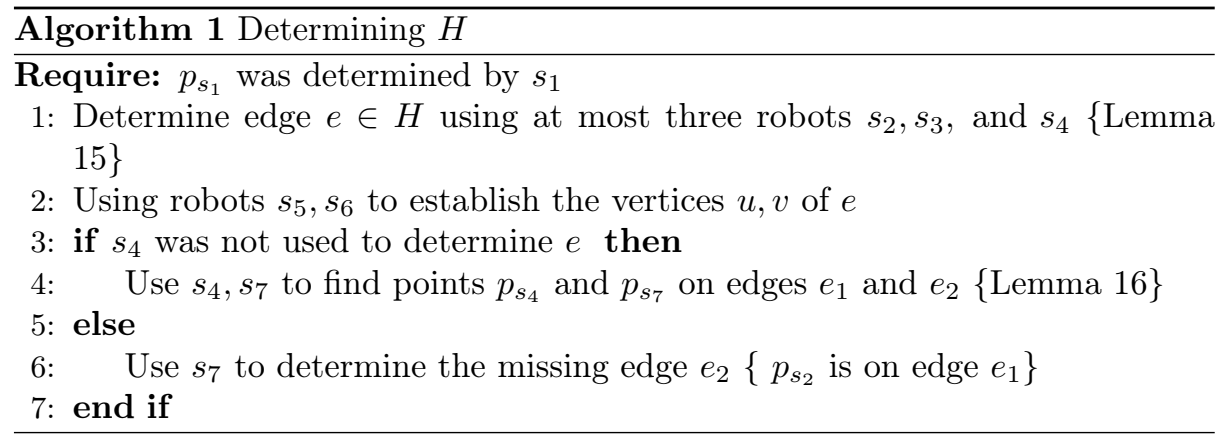

Given the restrictions on the edge of $H$, the robots use $m_{1} * \cos \left(15^{\circ}\right)$ as the width of vertical slabs and the height of the horizontal slabs to to construct path $\pi_{v h}$.

\subsection{Identifying $H$}

A high level algorithmic flow is given in Algorithm 1, where the details on how to accomplish its steps are given in the Lemmas.

The main idea behind the algorithm is to first identify an edge $e$ of $H$, including the two vertices $(u, v)$ of $e$. Once $e$ is determined then identify two more points on the boundary of $H$, one on each of the remaining edges leading to finding the location of $H$. The lemmas, which are identified beside the algorithms lines, present the required steps to complete the task.

In the poof of correctness we use the following notation, which are illustrated in Figure 11. Let $B$ be a box of size $\frac{m_{1}}{2} \times \frac{m_{1}}{2}$ centred at $p_{s_{1}}$ and let $B_{1}$ be a box of size $\frac{m_{1}}{4} \times \frac{m_{1}}{4}$ centred at $p_{s_{1}}$ where $p_{s_{1}}$ is the point where $s_{1}$ encountered $H$. Let $L_{s_{1}}$ be the line containing the last path segment of $s_{1}$. Let $p_{b}$ and $p_{b_{1}}$ be the intersection points of $B$ and $B_{1}$ with the path that $s_{1}$ has taken and are closest to $s_{1}$ when travelling backwards along the path.

Lemma 14. Let $p_{s_{1}}$ be the point encountered by $s_{1}$, and let $p_{s_{2}}$ be the point encountered by $s_{2}$ when travelling along Box B in clockwise direction. There can be at most one vertex of $H$ in the pocket formed by: path of $s_{1}$ between $p_{s_{1}}$ and $p_{b}$, the path of $s_{2}$ between $p_{b}$ and $p_{s_{2}}$, and the line segment $\left(p_{s_{1}}, p_{s_{2}}\right)$. 


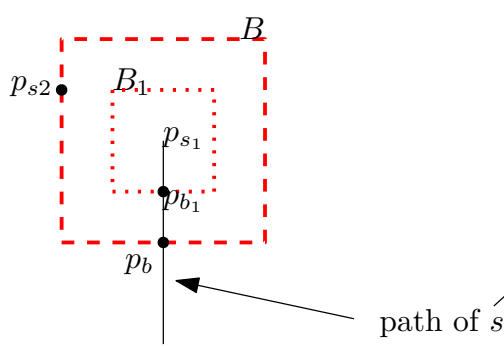

a.

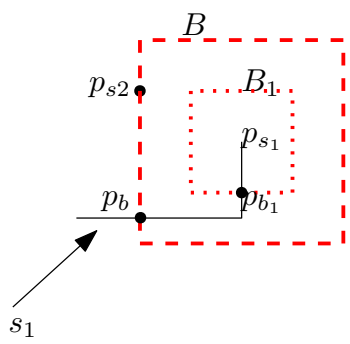

b.

Fig. 11. Point $p_{s_{1}}$ and the two boxes that are centred at it - box $B$ of size $\frac{m_{1}}{2} \times \frac{m_{1}}{2}$ and box $B_{1}$ of size $\frac{m_{1}}{4} \times \frac{m_{1}}{4}$. Examples of the intersection points $p_{b}$ and $p_{b_{1}}$ between the path taken by $s_{1}$ with boxes $B$ and $B_{1}$ respectively are shown in a. and b.

Proof. A box of size $\frac{m_{1}}{2}$ has a diameter of $\sqrt{\frac{m_{1}^{2}}{2}}<0.8 m_{1}$. Since the size of each edge of $H$ is at least $m_{1}$ at most one vertex of $H$ can be in side a box of size $\frac{m_{1}}{2} \times \frac{m_{1}}{2}$ centred at $p_{s_{1}}$.

Lemma 15. An edge e of $H$, where $p_{s_{1}} \in e$ can be determined using at most three additional robots $s_{2}, s_{3}$, and $s_{4}$.

Proof. When robot $s_{1}$ encounters $H$ for the first time an anchor point $p_{s_{1}}$ is established, which can either be a point on an edge or a vertex of $H$. Robot $s_{2}$ travels along the boundary of $B$ in clockwise direction, starting from the intersection of $B$ and the path of $s_{1}$, until it encounters $H$ at $p_{s_{2}}$. Next it is requires to establish whether the segment $\left(p_{s_{1}}, p_{s_{2}}\right)$ is on an edge of $H$.

Robot $s_{3}$ then travels along the boundary of $B_{1}$ in clockwise direction, starting $p_{b_{1}}$ until it encounters $H$ at $p_{s_{3}}$. There are two cases to examine depending on whether $p_{s_{1}}, p_{s_{2}}$, and $p_{s_{3}}$ are collinear.

1. $p_{s_{1}}, p_{s_{2}}$, and $p_{s_{3}}$ are collinear - in this case and edge $e$ was found using three robots

2. $p_{s_{2}}, p_{s_{1}}$, and $p_{s_{3}}$ are not collinear collinear - in this case, based on Lemma 14. there is at most one vertex in the pocket formed by the of $s_{1}$ between $p_{s_{1}}$ and $p_{b}$, the path of $s_{2}$ between $p_{b}$ and $p_{s_{2}}$ and the line segment $\left(p_{s_{1}}, p_{s_{2}}\right)$. Note that if $p_{s_{3}}$ is on a vertex of $H$ then $p_{s_{1}}$ and $p_{s_{3}}$ are on one edge of $H$ and $p_{s_{2}}$ and $p_{s_{3}}$ are on another edge of $H$. In order to determine whether $e$ is formed by $p_{s_{1}}$ and $p_{s_{3}}, s_{4}$ is travelling in a counter clockwise direction, starting at $p_{b_{1}}$ along the boundary of $B_{1}$ until it encounters $H$. Here two sub cases may arise:

(a) $p_{s_{1}}, p_{s_{3}}$ and $p_{s_{4}}$ are collinear - in this case, edge $e$ is found and $p_{s_{2}}$ is on a different edge of $H$.

(b) $p_{s_{1}}, p_{s_{3}}$ and $p_{s_{4}}$ are not collinear - in this case two edge of $H$ are determined - one by $p_{s_{2}}$ and $p_{s_{3}}$ and one by $p_{s_{1}}$ and $p_{s_{4}}$.

Once an edge $e$ was identified then either only the orientation of $e$ is known or the orientation of $e$ and additional point on one of the adjacent edges of $e$ are known. This depends on the number of robots that were used to determine the 
orientation of $e$. Thus, using at most two additional robots the end points of $e$ can easily be determined.

Lemma 16. Given a triangular HR $H$, seven robots are required, in the worst case to, determine the area covered by $H$.

Proof. Based on Lemma 15 there are two cases to examine:

1. Four robots, $s_{1}, s_{2}, s_{3}, s_{4}$, identified $e$ : in this case, based on the proof of Lemma 15, points $p_{s_{1}}$ and $p_{s_{4}}$ determine the orientation of $e$. Based, on Corollary 3. two more robots, $s_{5}$ and $s_{6}$, are required to identify the end points $(u, v)$ of $e$. Since $p_{s_{2}}$ is already on an edge, e.g., $e_{1}$, then one additional robot, $s_{7}$ is required to determine the remaining edge, $e_{2}$. Without loss of generality, let $v$ be the endpoint of $e$ that is incident to $e_{2}$. Robot, $s_{7}$, easily accomplish the task of identifying a point $p_{s_{7}}$ on $e_{2}$ by travelling around $v$ in a counter clockwise direction along the boundary of a box $B$ of size $\frac{m_{1}}{2} \times \frac{m_{1}}{2}$ centred at $v$.

The intersection point of the half lines $\overrightarrow{u p_{s_{2}}}$ and $\underset{v p_{s_{7}}}{2}$ determines the last vertex of $H$ and the extents of $e_{1}$ and $e_{2}$.

2. Three robots, $s_{1}, s_{2}, s_{3}$, identified $e$ : in this case $s_{1}, s_{2}$ and $s_{3}$ were collinear. Thus, the orientation of $H$ is known. Based, on Corollary 3 two more robots, e.g., $s_{5}$ and $s_{6}$, are required to identify the end points $(u, v)$ of $e$. Without loss of generality let $u$ be the rightmost vertex of $e$ when looking at $e$ from a point inside $H$ and that edge $e_{1}$ is incident to $u$ and $e_{2}$ is incident to $v$.

The remaining two edges, $e_{1}$ and $e_{2}$ of $H$ are determined by finding two points, one on each edge as follows: a. robot $s_{4}$ identifies a point $p_{s_{4}}$ on $e_{1}$ by travelling around $u$ in a clockwise direction along the boundary of a box $B$ of size $\frac{m_{1}}{2} x \frac{m_{1}}{2}$ centred at $u$; and b. robot $s_{7}$ identifies a point $p_{s_{7}}$ on $e_{2}$ by travelling around $v$ in a counter clockwise direction along the boundary of a box $B$ of size $\frac{m_{1}}{2} x \frac{m_{1}}{2}$ centred at $v$.

The intersection point of the half lines $\overrightarrow{u p_{s_{4}}}$ and $\overrightarrow{v p_{s_{7}}}$ determines the last vertex of $H$ and the extents of $e_{1}$ and $e_{2}$.

The following theorem summarized the result of Algorithm 1

Theorem 4. Given a triangular $H R H$ positioned in a rectangular region $P$, where $\forall e \in H, m_{1} \leq|e| \leq m_{2}$, a set of seven robots can determine the existence of $H$ and if $H$ exists can identify the area covered by $H$.

Algorithm 1 and Theorem 4 can easily be extended from triangular HRs to convex HRs with $n$ edges, where $\forall e \in H, m_{1} \leq|e| \leq m_{2}$, in a rectangular region $P$.

Theorem 5. Given a convex $H R H$ with $n$ edges positioned in a rectangular region $P$, where $\forall e \in H, m_{1} \leq|e| \leq m_{2}$, a set of $2 n+1$ robots can determine the existence of $H$ and if $H$ exists can identify the area covered by $H$. 


\section{Conclusion}

In this paper we introduce a new problem of detecting and identifying the location of hazardous entities in two dimensional space by a set of robots. If such a hazardous entity exists then find the area covered by it. We presented algorithms and a number of search paths for detecting HRs that are line segments, circles, and triangles. In particular we were interested in minimizing the number of robots required to identify the $H R$ and the search path length. Other optimization problems, which were not presented here, are the length and the effort required by the robots to determine the area covered by the HR once it was detected.

We showed that three or four robots are required when the $H R$ is a line segment, two or three robots are required when the $H R$ is circular, seven robots are required when the $H R$ is a triangle. We generalized the solution for a triangle to convex shapes and show that $2 n+1$ robots are required when the $\mathrm{HR}$ is a convex shape.

A number of open problems arise: Can a lower bound be found for the detection problem of different shapes? We are also investigating the increased complexity when more than one hazardous entity may be located in the region. An interesting and harder problems is when the HR is not stationary. For example, what are the strategies and the required number of robots when the hazardous entity is moving (e.g., at half the speed of the robots).

\section{References}

1. Chen, A., Kumar, S., Lai, T.H.: Designing localized algorithms for barrier coverage. In: Proceedings of the 13th annual ACM international conference on Mobile computing and networking. pp. 63-74. MobiCom '07, ACM, New York, NY, USA (2007). https://doi.org/10.1145/1287853.1287862 http://doi.acm.org/10.1145/ 1287853.1287862

2. Cole, R., Yap, C.K.: Shape from probing. Journal of Algorithms 8(1), $19-38$ (1987)

3. Collins, A., Czyzowicz, J., Gasieniec, L., Kosowski, A., Kranakis, E., Krizanc, D., Martin, R., Morales Ponce, O.: Optimal patrolling of fragmented boundaries. In: Proceedings of the 25th ACM symposium on Parallelism in algorithms and architectures. pp. 241-250. SPAA '13, ACM, New York, NY, USA (2013). https://doi.org/10.1145/2486159.2486176, http://doi.acm.org/10.1145/ 2486159.2486176

4. Czyzowicz, J., Kowalski, D., Markou, E., Pelc, A.: Complexity of searching for a black hole. Fundamenta Informaticae 71(2-3), 229-242 (2006), www.scopus.com, cited By (since 1996):17

5. Dobrev, S., Flocchini, P., Prencipe, G., Santoro, N.: Mobile search for a black hole in an anonymous ring. Algorithmica (New York) 48(1), 67-90 (2007), www . scopus. com

6. Flocchini, P., Kellett, M., Mason, P.C., Santoro, N.: Searching for black holes in subways. Theor. Comp. Sys. 50(1), 158-184 (Jan 2012). https://doi.org/10.1007/s00224-011-9341-8, http://dx.doi.org/10.1007/ s00224-011-9341-8 
7. Guibas, L.J., claude Latombe, J., Lavalle, S.M., Lin, D., Motwani, R.: A visibilitybased pursuit-evasion problem. International Journal of Computational Geometry and Applications 9, 471-494 (1996)

8. Kruskal, J.: On the shortest spanning subtree of a graph and the traveling salesman problem. Proceedings of the American Mathematical Society 7(1), 48-50 (1956)

9. Nussbaum, D., Thoumy, S.: Identifying hazardous shapes in the plane. In: Proceedings of the 3rd IEEE International Conference on Robotic Computing. pp. 271-276. IRC 2019, Napoli, Italy (2019)

10. O'Rourke, J.: Art Gallery Theorems and Algorithms. The International Series of Monographs on Computer Science, Oxford University Press, New York, NY (1987)

11. Skiena, S.: Problems in geometric probing. Algorithmica 4(1), 599-605 (1989), www.scopus.com, cited By (since 1996):16 